\title{
Molecular dynamics simulation of gold cluster growth during sputter deposition
}

\author{
J. W. Abraham, ${ }^{1}$ T. Strunskus, ${ }^{2}$ F. Faupel, ${ }^{2}$ and M. Bonitz ${ }^{1, *}$ \\ ${ }^{1}$ Institut für Theoretische Physik und Astrophysik, Christian-Albrechts-Universität \\ zu Kiel, Leibnizstraße 15, D-24098 Kiel, Germany \\ ${ }^{2}$ Institut für Materialwissenschaft, Lehrstuhl für Materialverbunde, \\ Christian-Albrechts-Universität zu Kiel, Kaiserstraße 2, D-24143 Kiel, Germany
}

(Dated: June 14, 2021)

\begin{abstract}
We present a molecular dynamics simulation scheme that we apply to study the time evolution of the self-organized growth process of metal cluster assemblies formed by sputter-deposited gold atoms on a planar surface. The simulation model incorporates the characteristics of the plasma-assisted deposition process and allows for an investigation over a wide range of deposition parameters. It is used to obtain data for the cluster properties which can directly be compared to recently published experimental data for gold on polystyrene (M. Schwartzkopf et al., ACS Appl. Mater. Interfaces 7, 13547 (2015)). While good agreement is found between the two, the simulations additionally provide valuable time-dependent real-space data of the surface morphology some of whose details are hidden in the reciprocal-space scattering images that were used for the experimental analysis.
\end{abstract}

Keywords: metal-polymer nanocomposites, sputter deposition, molecular dynamics simulation, gold, polystyrene

\section{INTRODUCTION}

Nanocomposites have been subject to extensive experimental and theoretical research in the last decades because the assembly of materials with contrasting properties may result in remarkable characteristics of the composite. The variety of technological applications based on nanocomposites can be found in, e.g., electronics [1-4], plasmonics [5, 6], food packaging [7] and medicine [8, 9]. While various types of materials are present in synthetic and natural nanocomposites, metal-polymer nanocomposites are of specific interest as they are promising candidates for the fabrication of materials with tailored magnetic, electronic and optical properties [10 16] at low cost.

Typical physical vapor deposition processes of metalpolymer nanocomposites comprise simultaneous deposition of metal and polymer, leading to nanoparticles embedded in a polymer host matrix, or exclusive deposition of metal atoms or clusters onto a prepared polymeric thin film, restricting the nanoparticle formation to the surface and near-surface area of the polymer bulk [15, 17 17 20. Depending on the desired properties of the composite, different deposition methods of both polymer and metal may be advantageous [18, 21, 24. For example, sputter deposition of metal atoms allows for high deposition rates, but the employed plasma also affects the surface structure (see below). While many of the experimental approaches have the common goal of getting independent control over sizes, shapes and spatial distributions of the nanoparticles, they also share the difficulty that the selforganized nanoparticle formation process can only be influenced indirectly, e. g., by changing deposition rates or substituting materials. Therefore, in situ observations

\footnotetext{
* mail to: bonitz@theo-physik.uni-kiel.de
}

of the film characteristics during the deposition process are useful to deepen the understanding of the relevant growth kinetics.

Recent progress in experimental monitoring of film growth was made by applying grazing incidence smallangle X-ray scattering (GISAXS) to trace the timeresolved morphology of sputter-deposited gold on polymer surfaces. [14, 20, 25]. This experimental method relies on extracting all structural information from the features in the scattering patterns. GISAXS experiments are well suited to measure the evolution of mean cluster properties such as radii, heights and distances between clusters, but they lack the possibility to observe the atomic structure of individual clusters in real space. Furthermore, it is impossible to capture the microscopic physical processes that drive the cluster formation. These processes comprise thermally activated diffusion of metal atoms on the surface, desorption of single atoms with high kinetic energy, coalescence of metal clusters, and direct attachment of deposited atoms to existing clusters. At that, the interaction between metal atoms is typically much stronger than that between metal atoms and the polymer. Furthermore, the plasma which is applied to sputter atoms from the metallic target also influences the cluster growth. Not only does it transfer charges to the nanoparticles, by ejecting highly energetic ions in the direction of the substrate it also causes defects at the surface, where the trapping probability of metal atoms is strongly increased [26, 27].

One possibility to improve the understanding of the complex interplay of the mentioned processes is offered by computer simulations. So far, however, we are not aware of any computational studies of thin-film growth by sputter deposition which resolve not only the atomic structure of the clusters, but also take into account the influence of the polymer and the plasma background. Although kinetic Monte Carlo simulations have proved to be appropriate for the description of similar systems, 
they are usually restricted to cases with simple cluster geometries that allow one to neglect the motion of individual atoms such that clusters can be approximated by simple geometrical objects, e.g., spheres or columnar structures [16, 28, 29. In this work, we go beyond these limitations by introducing an atomic scale molecular dynamics (MD) simulation scheme for the motion of sputter-deposited gold particles onto a polymeric surface. Although the incorporation of individual atoms is on the cost of accessible system sizes, the method allows us to perform computations with surface sizes of at least $45 \mathrm{~nm} \times 45 \mathrm{~nm}$. As we will show in a parameter study for film thicknesses up to $3 \mathrm{~nm}$, the simulated systems are big enough to directly compare and find agreement with recently published experimental data for sputtered gold $(\mathrm{Au})$ on a polystyrene (PS) film [20]. Furthermore, the employed parameters and potentials can easily be adjusted in order to represent different plasma conditions or materials.

Although molecular dynamics has been a successful and established method to simulate the deposition of thin films for several decades, e. g., 30 35, our work attains distinction through the incorporation of the characteristics of sputter-deposited thin metal films as well as the possibility to perform close comparisons with experimental data. In the following, we briefly explain in what aspects our simulation method differs from other common approaches.

First, the treatment of the polymer surface is not particle-based. Instead the diffusive motion of the metal atoms on the surface is driven by fluctuating and dissipative forces. This is much simpler than atomistic or coarsegrained surface models, but it has been shown in previous kinetic Monte Carlo (KMC) studies [16, 17, 29, 36, 37. that the diffusive motion of the metal atoms can be appropriately described by employing a continuum model for the polymer. This treatment is physically motivated by the facts that the assembly of polymer chains is very disordered and the interaction between metal and polymer is typically very weak [38. The stochastic description of the atomic motion allows us to set diffusion coefficients and spend the saved computer resources on the simulation of more metal atoms. For example, the number of particles in our simulations is about two orders of magnitude larger than typical numbers in simulations with particle-based substrates [34, 35]. Another advantage of a tunable diffusion coefficient is that it is straightforward to obtain realistic values of the distance that a diffusing atom travels during the average time between the deposition of two particles. In a particle-based model of the polymer surface, this would require a more complicated modification of the heat bath or the potentials of the cross interaction between metal and polymer particles.

As a second difference, we implemented some idealized model processes in order to mimic the re-evaporation of atoms from the surface and the creation of surface defects. Although these processes do not represent the ex-

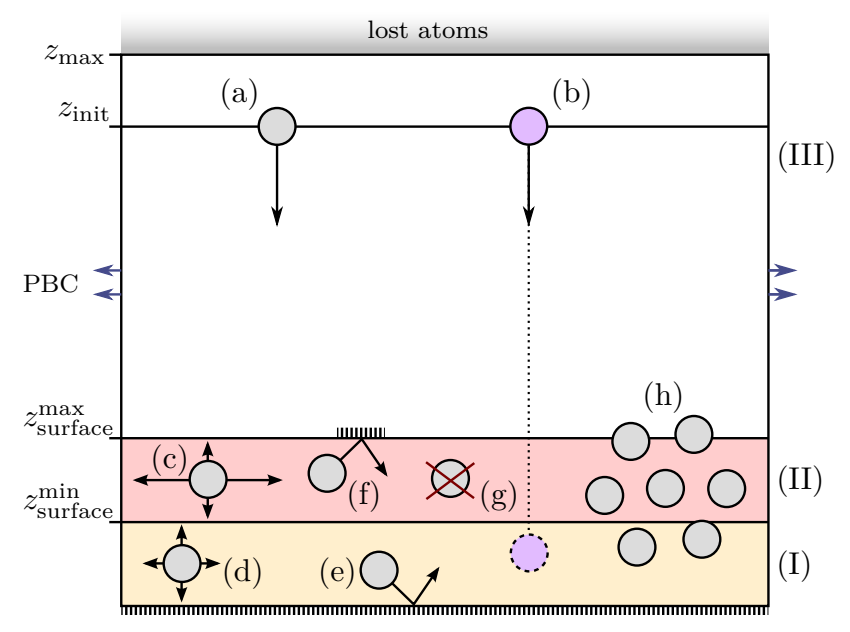

FIG. 1. Illustration of atomic processes in different parts of the simulation box (side view): deposition of atoms (a), deposition and implantation of ions (b), diffusion in the surface layer (c) and in the bulk layer (d), reflection of atoms at the bottom wall of the simulation box (e), reflection of neighborless atoms at the top of the surface (f), removal of atoms from the surface due to re-evaporation $(\mathrm{g})$, cluster formation and crossing of the border at $z_{\text {surface }}^{\max }(\mathrm{h})$. The layers correspond to the polymer bulk (I), the surface (II) and the plasma environment above the surface (III). While the simulation box has periodic boundary conditions (PBC) in horizontal directions, atoms that dissociate from clusters and small clusters that very rarely dissociate from the surface are removed from the simulation after crossing the top border of the simulation box.

act atomistic behavior of the system, they enable us to make statements about the qualitative influence of the corresponding real physical processes, and they offer the advantage of being easily adjustable to specific conditions. Hence, we combine the advantages of a microscopic treatment, which is applied in standard molecular dynamics, and the introduction of simplified model processes, which is typical for kinetic Monte Carlo simulations [39].

Further details of the simulation scheme and its relation to realistic experimental scenarios are explained in Sec. III. Following this, in Sec. III, a comparison with experimental data and a parameter study of cluster properties for various trapping parameters, re-evaporation rates and deposition rates are presented.

\section{SIMULATION SCHEME}

All simulations were carried out using the $L A M M P S$ software package 40. The code performs standard molecular dynamics simulations with specific boundary conditions and additional processes for the addition and removal of atoms. For all particles, the interatomic interaction potential is calculated with the embedded-atom method [41, using tabulated data for gold provided by 
the work of Foiles at al. 42. In the following, we describe the partitioning of the simulation box and the specific treatment of the particles.

\section{A. Details of the model}

As illustrated in Fig. 1, the simulation box with the dimensions $\left[0, x_{\max }\right],\left[0, y_{\max }\right],\left[0, z_{\max }\right]$ and periodic boundary conditions in $x$ - $y$-directions is divided into different layers that are intended to represent the behavior of the particles above the surface $\left(z>z_{\text {surface }}^{\max }\right)$, on the surface $\left(z_{\text {surface }}^{\min }<z \leq z_{\text {surface }}^{\max }\right)$ and in the uppermost part of the polymer bulk $\left(0<z \leq z_{\text {surface }}^{\min }\right)$. We stress that the surface layer has a finite thickness in order to take into account the roughness of the polymer surface.

For all simulation results presented in this work, we used the values $x_{\max }=y_{\max }=45 \mathrm{~nm}, z_{\max }=8 \mathrm{~nm}$, $z_{\text {surface }}^{\min }=0.2 \mathrm{~nm}$ and $z_{\text {surface }}^{\max }=0.6 \mathrm{~nm}$. As mentioned before, the dynamics of individual building blocks of the polymer chains are ignored in this model. Instead, the polymer is treated as a continuous fluid-like background to the gold particles, enabling us to perform Langevin dynamics simulations. In the subsequent paragraphs, we address further details of the simulation process.

a. Iteration cycle All quantities of interest are monitored after each iteration period consisting of $N_{\text {iter }}=300$ steps. This involves updates of the associated layers and calculations of the coordination number to identify atoms on the surface without neighbors.

b. Creation of particles It is not necessary to simulate the processes in the $\mathrm{Au}$ cathode that lead to the ejection of atoms. Instead, we assume that there is a constant flux of sputtered atoms and ions that travel towards the surface. We model this by continually creating atoms and ions at random positions in the plane at $z_{\text {init }}=7 \mathrm{~nm}$ (cf. Fig. 1) with the initial velocity $\mathbf{v}_{\text {init }}=\left(0,0,-\left|v_{\text {init }}\right|=-0.1 \mathrm{~nm} / \mathrm{ps}\right)$ [4]. The time between particle creation events can be calculated from the deposition rate $J$.

c. Motion of the particles With the exception of the space between $z_{\text {surface }}^{\max }$ and $z_{\max }$, where the motion of the metal particles is treated microcanonically, the diffusive motion on the surface is modeled by friction and fluctuating forces using Langevin dynamics [44. Thus we assume that the polymer acts like a continuous heat bath in thermal equilibrium. The corresponding equation of motion for all particles with the coordinates $\mathbf{r}=\left(\mathbf{r}_{1}, \mathbf{r}_{2}, \ldots\right)$ reads

$$
m \ddot{\mathbf{r}}=-\nabla U(\mathbf{r})-\frac{m}{t_{\mathrm{damp}}} \dot{\mathbf{r}}+\mathbf{F}_{\text {fluct }},
$$

where $m$ is the mass of a gold atom, $t_{\text {damp }}$ controls the strength of the friction, and $U$ is the interatomic potential. The fluctuating force obeys the proportionality

$$
\mathbf{F}_{\text {fluct }} \propto \sqrt{\frac{k_{\mathrm{B}} T m}{t_{\mathrm{damp}} \Delta t}},
$$

where $T$ is the substrate temperature, $k_{\mathrm{B}}$ is the Boltzmann constant, and $\Delta t$ is the timestep. The direction and the magnitude of $\mathbf{F}_{\text {fluct }}$ are randomized according to Ref. [45].

d. Diffusion Metal atoms on polymer substrates are known to not only diffuse on the surface, but also to penetrate slowly into the bulk. The application of Langevin dynamics allows us to simulate such an anisotropic diffusive motion by adjusting the damping parameters $t_{\text {damp }}$ for specific directions and areas of the simulation box. The corresponding diffusion coefficients can be calculated via

$$
D=\frac{1}{m} k_{\mathrm{B}} T t_{\mathrm{damp}} .
$$

In the surface layer, the diffusion coefficient for the horizontal motion, $D_{\text {surface }}^{\|}$, is assigned a value that is bigger than the one for the vertical motion, $D_{\text {surface }}^{\perp}$. For the bulk layer, we set $D_{\text {bulk }}^{x / y / z}=D_{\text {surface }}^{\perp}$, i. e., we keep the diffusion coefficient of the vertical motion in the surface layer and assign it to all directions. As it is stated in Ref. [36] that the ratio

$$
\alpha=\frac{D_{\text {surface }}^{\|}}{D_{\text {bulk }}^{x / y / z}} .
$$

is presumably larger than 60 , we performed all simulations with $\alpha=80$. This choice is rather arbitrary, but we carefully verified that the results do not show significant differences for ratios as low as $\alpha=30$. Furthermore, the value can easily be adjusted once it has been determined for specific materials in external studies. In addition to the reduction of the bulk diffusion by decreasing the corresponding diffusion coefficients $D_{\text {bulk }}^{x / y / z}$ and $D_{\text {surface }}^{\perp}$, all atoms are reflected at the bottom of the simulation box. This is motivated by the fact that deep penetration into the bulk is not observed in the experiments from Ref. [20]. However, we remark that there exist other experimental situations which require an accurate description of clusters diffusing in the polymer bulk [19].

e. Re-evaporation The condensation coefficient $C$ of metal on metal is close to one due to the strong metallic bonds. Hence, re-evaporation of atoms from clusters is rarely observed in experiments. In the simulations, the employed potential ensures that this behavior is reproduced. In contrast, the condensation coefficient of metals on polymers at room temperature strongly depends on the involved materials. For example, the values $C=0.006$, for $\mathrm{Au}$ on Teflon $\mathrm{AF}$, and $C=0.955$, for $\mathrm{Au}$ on PMDA-ODA polyimide, were reported 46, 47. Consequently, re-evaporation of metal atoms from the surface has to be considered in the simulations. In fact, if we performed pure Langevin dynamics in the surface layer, some free atoms would occasionally cross the border at $z_{\text {surface }}^{\max }$ and then be likely to reach the top of the simulation box and get lost. However, this behavior does not correspond to the actual physical process 
and it cannot be controlled without changing other important model parameters. We therefore implement another simple model process that allows us to take into account the desorption of single metal atoms. For that purpose, we force all free metal atoms to remain in the two bottom layers by defining another reflective boundary at $z_{\text {surface }}^{\max }$. This boundary only acts on atoms that have no neighbors within the cutoff distance of the interaction potential, i. e., atoms belonging to a cluster are not affected by this modification. In a next step, we define a process that removes neighborless atoms from the surface layer with a probability $p_{\text {re }}$ within each iteration cycle. This probability can be understood as a parameter that mimics the characteristic sticking behavior of a specific material.

f. Lost atoms Atoms that belong to clusters $\left(N_{\text {atoms }}>1\right)$ are allowed to cross the boundary at $z_{\text {surface }}^{\max }$. Due to the temperature-induced fluctuating forces, there is a very small, but finite probability that atoms escape from a cluster. Most of the time, these atoms travel in a straight line towards the top of the simulation box and are removed once they reach $z_{\max }$.

g. Trapping of atoms A certain fraction of the gold atoms adheres to the surface immediately or shortly after they reach the surface in an actual experimental set-up. There are several reasons for this behavior. On the one hand, the untreated polymer surface exhibits some sites with increased trapping probabilities due to its unevenness and imperfections. Furthermore, during the deposition process, additional surface defects are created by the impingement of highly energetic ions from the plasma, e.g., $\mathrm{Ar}^{+}$if an Argon plasma is used. On the other hand, knowing that typical energy distributions of sputtered metal particles have a long tail that exceeds several tens of $\mathrm{eV}$ 48, one can expect that some of the sputtered $\mathrm{Au}$ atoms are sufficiently fast for being implanted into the polymer bulk. Since there do not exist detailed microscopical studies of these effects, neither the sizes nor the number of surface defects are known. For that reason, we restrict ourselves to simulating the qualitative behavior of the surface defects with a specific treatment for a fraction $\gamma$ of all deposited particles. If one of these particles reaches a point below $z_{\text {surface }}^{\mathrm{min}}$, its position is kept fixed, but the interaction with the other atoms is maintained. If instead the particle is deposited onto other metal atoms and thus does not reach the surface layer, it is treated just like the other atoms.

h. Adjustment to experimental parameters In the experimental set-up presented in Ref. 20, the deposition rate $J_{\exp }=0.49 \mathrm{~nm} / \mathrm{min}$ and the surface diffusion coefficient $D_{\text {exp }}=7.33 \times 10^{-18} \mathrm{~m}^{2} / \mathrm{s}$ were measured. From the knowledge of $J_{\text {exp }}$, one can calculate the average time between the deposition of two gold atoms per $\mathrm{nm}^{2}$, which is about $2 \mathrm{~s}$. At the same time, the mean squared displacement of normal two-dimensional diffusive motion can be used to estimate the distance that an atom travels

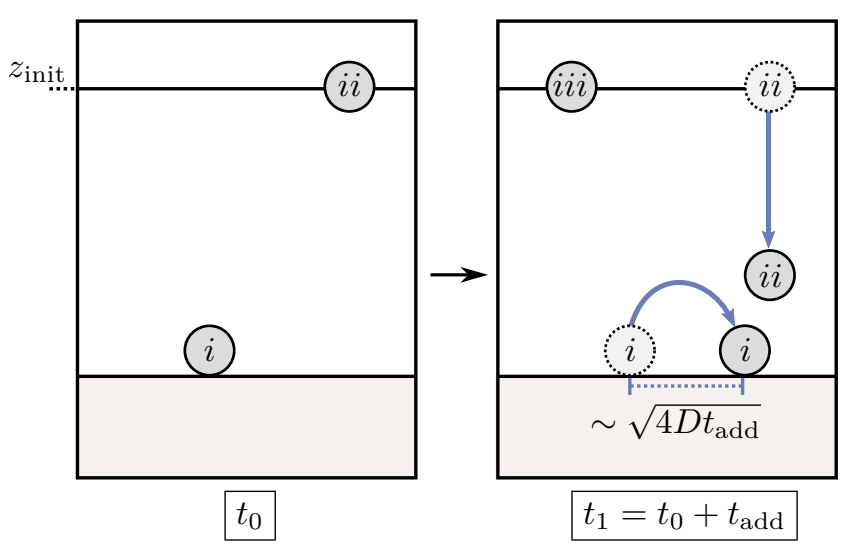

FIG. 2. Principle of the proportional rescaling of the deposition rate $J$ and the diffusion coefficient $D$. For all combinations of $J$ and $D$ with $J / D=$ const., the mean squared displacement of particle $i$ is preserved during the time $t_{\text {add }}$ that it takes to create particles $i i$ and $i i$ at random positions in the plane at $z_{\text {init }}$.

during the time $t$, according to

$$
l:=\sqrt{(r(t)-r(0))^{2}}=\sqrt{4 D_{\exp } t} .
$$

For $l=1 \mathrm{~nm}$, we obtain $t=0.03 \mathrm{~s}$. These values indicate that the deposition process and the diffusive motion of the particles are many orders of magnitude slower than the cluster formation processes, which happen on time scales of pico- and nanoseconds. In order to capture these fast processes, we set the timestep to $\Delta t=0.001 \mathrm{ps}$. As the number of timesteps to obtain a sufficiently large system must not exceed a practical limit, this choice requires that the simulations are performed with very high deposition rates $J$ and diffusion coefficients $D_{\text {surface }}^{\|}$, which considerably outreach typical experimental values. This is a common problem in MD simulations of deposition processes. For example, in Ref. 31] thermal activated diffusion during the time between arriving atoms is neglected. Another approach is to switch between NVE and NVT ensembles to make sure that the system relaxes after each deposition event 49. Since the present model treats diffusion via Langevin dynamics, it is possible to preserve a realistic value for the mean squared displacement of neighborless particles during the time between two deposition events. This can be done by performing the simulations with proportionally increased experimental values of the deposition rate and the diffusion coefficient. Hence, by maintaining the ratio

$$
\xi:=\frac{D_{\text {surface }}^{\|}}{D_{\exp }}=\frac{J}{J_{\exp }}
$$

in the simulations, the mean squared displacement of a diffusing particle, $l^{2} \propto D t$, is preserved because both the diffusion coefficient and the time enter linearly. An illustration of this principle can be found in Fig. 2, which 
displays an exemplary motion of a single particle on the surface during the time $t_{\text {add }} \propto J^{-1}$ between the deposition of two other particles. In practice, we first define $D_{\text {surface }}^{\|}$according to Eq. (3) by setting the substrate temperature to the experimental value $T=296 \mathrm{~K}$ and choosing the value $t_{\text {damp }}^{\text {surface } \|}=1 \mathrm{ps}$ for the damping parameter. Afterwards, we take the values for $D_{\exp }$ and $J_{\exp }$ from Ref. 20] to calculate the ratio $\xi$ and finally the deposition rate $J$. For the mentioned parameters, we obtain the values $1.2 \mathrm{~ns}$ for the average time between the deposition of two atoms per $\mathrm{nm}^{2}$ and $20 \mathrm{ps}$ for the time a diffusing atom to reach a mean squared displacement of $1 \mathrm{~nm}^{2}$.

We remark that the employment of Langevin dynamics, which treats all atoms on the surface and in the bulk as if they are surrounded by a continuous fluid, is only an approximation of the realistic diffusion process, which is mainly caused by the interaction between the fluctuating molecules of the polymer chain and the atoms at the outer cluster shell. This is also the reason for the fact that one cannot make the simulations more realistic just by decreasing the damping parameter $t_{\text {damp }}^{\text {surface, } \|}$ because that would lead to strongly damped motion of all cluster atoms that are in the bulk or the surface layer. Although it is very difficult to associate our choice of $t_{\text {damp }}^{\text {surface, }}$ with specific materials, we made sure that the chosen parameter set enables fast formation, coalescence and equilibration of stable clusters in the simulations. Furthermore, we checked that despite the strongly increased deposition rate, nucleation does not happen in the gas phase, but only on the surface.

\section{RESULTS}

Before we present the simulation results in this section, we mention some details about the evaluation of the cluster properties. Furthermore, in order to show the limitations of the comparability between simulated and experimental results, we briefly explain how the experimental data were obtained in Ref. [20].

\section{A. Evaluation of simulation results}

In the following, we explain how we determine the cluster properties from the atom positions obtained in the simulations.

The first step of the evaluation is the identification of clusters. For that purpose, an atom is defined to belong to a cluster if its distance to at least one of the cluster atoms is below the threshold value $0.32 \mathrm{~nm}$. The employed algorithm was taken from the software OVITO [50]. Furthermore, we remark that we define that the minimum size of a cluster is 2 atoms, i.e., all neighborless atoms on or above the surface are excluded from the evaluation of the following quantities.
All presented cluster radii refer to the maximum of the horizontal distances of the cluster atoms and the center of mass of the cluster. In order to get reasonable values in the case of clusters that extend over one or more periodic images of the simulation box, we use the generalized method for the calculation of the center of mass in a system with periodic boundary conditions from Ref. [51. Of course, this definition of the radius is only a rough measure for the cluster extension in the case of clusters with complex, non-spherical shapes.

The cluster heights are characterized with the help of the distribution function of vertical atom positions, $f(z)$. In the results provided below, the heights are indicated by the values $\tilde{z}$ for which the cumulative distribution function,

$$
\int_{0}^{\tilde{z}} f(z) \mathrm{d} z
$$

reaches 0.99 , i. e., $99 \%$ of all atoms have a vertical position below $\tilde{z}$.

For the mean cluster distance $D$, we use the estimator

$$
D=\left(A / N_{\text {cluster }}\right)^{1 / 2}
$$

where $A$ is the surface area of the simulation box and $N_{\text {cluster }}$ is the total number of clusters on the surface. Similar to the cluster radius, this quantity is only meaningful as long as there is a large number of isolated homogeneously distributed clusters on the surface.

In addition to these cluster properties, we also provide results for the fraction of the surface that is covered with metal. After binning the atom position on a twodimensional grid which is parallel to the surface, the coverage is obtained by calculating the ratio of the number of occupied bins and the total number of bins. The side length of a bin is set to half the value of the lattice constant of Au. While maintaining a high resolution, this choice is big enough such that a homogeneous gold layer corresponds to a surface coverage of one.

Finally, we remark that we plot all quantities in dependence of the effective film thickness $\delta$ which is the height of a homogeneous metal film that contains as many atoms as can be found on the surface. We stress that this quantity is not necessarily proportional to the time because due to desorption effects, the number of atoms on the surface is usually less than the total number of deposited atoms.

\section{B. Experimental method}

Whereas the simulations provide atom positions in real space, in Ref. 20], the surface morphology was investigated in reciprocal space, using the GISAXS method [52, 53]. This method relies on the analysis of the angledependent intensity structure of scattered X-rays. Most cluster properties were obtained by projecting the scattered structures onto a geometrical model of uniform 


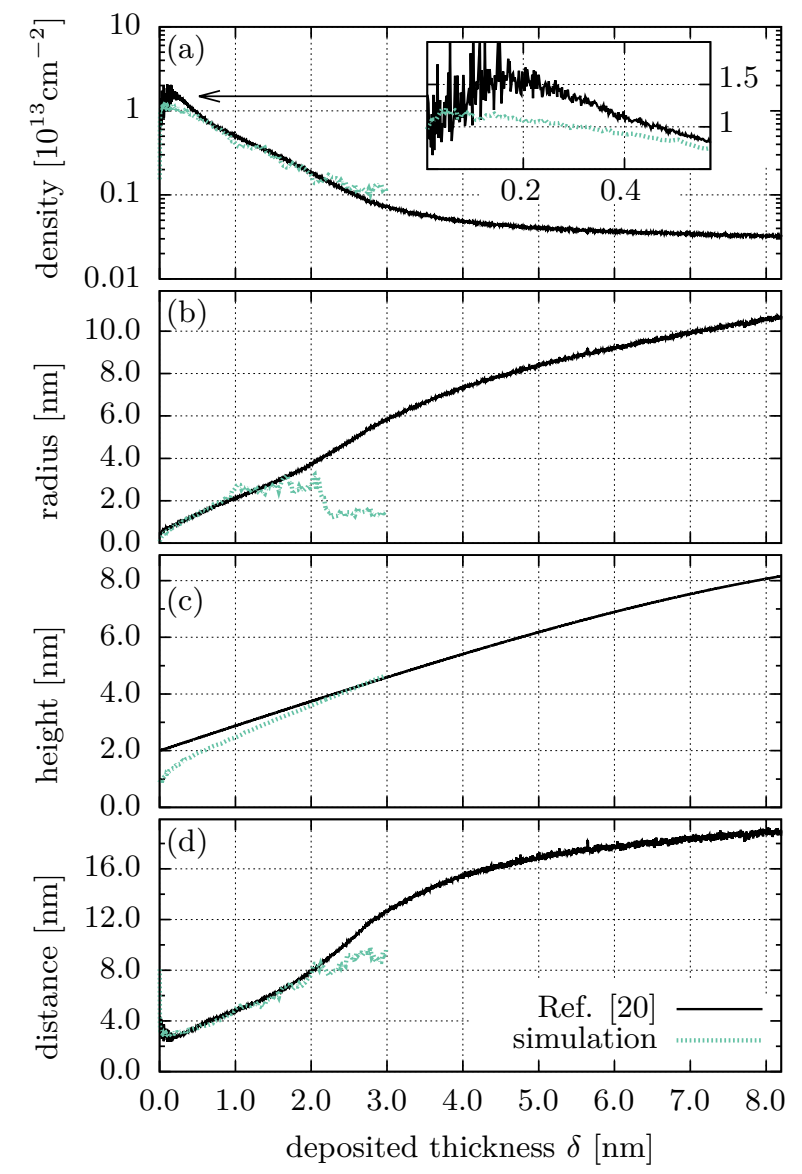

FIG. 3. Comparison of cluster properties as obtained from experimental results in Ref. 20] and MD simulations with selected values for the re-evaporation probability, $p_{\text {re }}=$ $1 \times 10^{-4}$, and the fraction of trapped Au particles, $\gamma=$ $1 \times 10^{-2}$. For deposited thicknesses $\delta$ less than about $2 \mathrm{~nm}$, all quantities agree with experimental trends. However, with the formation of a percolated network of non-spherical clusters for larger $\delta \gtrsim 2 \mathrm{~nm}$, the cluster radius and the cluster distance are no longer meaningful quantities.

hemispherical clusters distributed on a two-dimensional hexagonal lattice. The cluster distance $D$ was calculated according to $D=2 \pi / q_{0}$, where $q_{0}$ is the inverse length coordinate of the first lateral maximum of the scattered intensity. Similarly, the effective thickness of the film $\delta$ was determined from the vertical structure of the scattered intensity. Then, the cluster radius was calculated such that the volume of the hemispherical clusters is equal to the effective volume of a homogeneous layer with the thickness $\delta$. The cluster heights were independently determined by fitting a hemispherical cluster model to the measured GISAXS data with the software IsGISAXS [54. Hence, when comparing the simulated data to the experimental data, one has to keep in mind that some details of the local film structure vanish by the averaging procedure of generating a fit to simplified geometrical objects.

\section{General behavior of the system}

We start the presentation with a general comparison of experimental results from Ref. 20] and selected simulation results of a run with a computation time of several days on a computer cluster with $\sim 1000 \mathrm{CPU}$ cores. The corresponding simulation parameters are $p_{\text {re }}=1 \times 10^{-4}$ for the re-evaporation probability and $\gamma=1 \times 10^{-2}$ for the fraction of trapped Au particles. In Fig. 3, the corresponding cluster densities, radii, heights and distances are shown over the full range of experimentally obtained effective film thicknesses $\delta$. Whereas in the experiments, a film with the thickness $\delta=8 \mathrm{~nm}$ was deposited, we restricted the longest simulation run and most of the other runs to the deposition of a film with the thickness $\delta=3 \mathrm{~nm}$ and $\delta \approx 1 \mathrm{~nm}$, respectively. These restrictions of simulated thicknesses are imposed by the computational run time which scales according to $\mathcal{O}\left(N_{\text {atoms }}\right)$. With the given surface size, each deposited nanometer corresponds to $1.2 \times 10^{5}$ atoms. The simulation time depends on the values of $\gamma$ and $p_{\text {re }}$, because these parameters affect the sticking of atoms to the bare surface. While only $7.2 \times 10^{7}$ steps $(72 \mathrm{~ns})$ are required to deposit the first nanometer with the parameters $\gamma=5 \times 10^{-2}$ and $p_{\text {re }}=1 \times 10^{-4}$, it takes $18.7 \times 10^{7}$ steps (187 ns) to deposit the same thickness with the parameters $\gamma=1 \times 10^{-3}$ and $p_{\text {re }}=1 \times 10^{-1}$.

Although only the initial period of the film growth in strong non-equilibrium is simulated, the data is sufficient capture the non-monotonic behavior of the cluster density and the average cluster distance. In the following, we describe in detail how the cluster properties evolve. For a better understanding, we also refer to the snapshots of simulated cluster structures in Fig. 4, which serve as an illustration to the results shown in Fig. 3.

The number density of clusters shown in Fig. 3(a) exhibits a sharp maximum at $\delta \approx 0.1 \mathrm{~nm}$ followed by a nearly exponential decay that transitions into a saturated state beginning at $\delta \approx 5 \mathrm{~nm}$. The maximum originates from two processes with opposing effects: while the addition of atoms to the system leads to the formation of new clusters, other clusters coalesce and thus reduce the number of clusters. In the case of small clusters, the coalescence is mainly driven by the diffusive motion that the clusters perform along the surface. However, the more atoms a cluster contains, the slower this motion becomes. In this case, lateral aggregation is caused by the attachment of atoms and small clusters to existing clusters. Once a large fraction of the polymer surface is covered with metal, most incoming atoms are directly deposited onto an existing big cluster. Consequently, lateral growth becomes less important with increasing film thickness. This explains why the density only slowly changes for high values of $\delta$. Comparing experimental data and simulation results, we find partial quantitative agreement, in particular concerning the $\delta$-value of the maximum and the subsequent decaying values of the density. As we will show in the discussion below, qualitative agreement is 

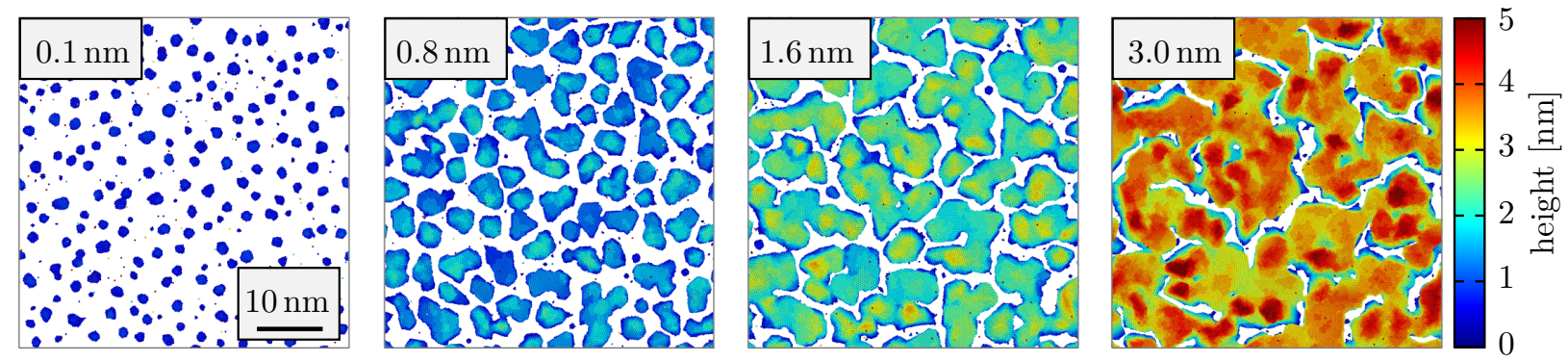

FIG. 4. Top view snapshots of the cluster morphology over a wide range of effective deposited thicknesses from $0.1 \mathrm{~nm}$ (18000 atoms) to $3 \mathrm{~nm}$ (357000 atoms). The figure illustrates the data set of a single run with the re-evaporation probability $p_{\text {re }}=1 \times 10^{-4}$, the fraction of trapped atoms $\gamma=1 \times 10^{-2}$ and the surface length $45 \mathrm{~nm}$. The colors indicate the vertical position of the atoms.

found for many parameter sets, but - especially near the position of the maximum density - the numerical data is very sensitive to simulation parameters.

In Fig. 3(b), the cluster radii can be compared. While the experimental curve does not display any deviation from the monotonic growth behavior, the simulation results start fluctuating for $\delta>1 \mathrm{~nm}$. This behavior is attributed to the aforementioned non-spherical cluster shapes and poor statistics due to the finite size of the simulation box. For $\delta>2 \mathrm{~nm}$, there is even a drop of the curve. The reason for this is the co-existence of few large cluster structures and several very small clusters that all contribute with the same weight in the averaging procedure to calculate the mean radius. These fluctuations do not occur in the experimental data, because the monodisperse cluster model is also used for elongated cluster structures. Consequently, the values we obtained for the radii are only meaningful for thicknesses up to $\delta \approx 1 \mathrm{~nm}$. In this regime, we find indeed good agreement with experimental data.

Similar to the cluster radii, the cluster heights shown in Fig. 3(c) are also characterized by a monotonic growth. Since the film becomes more dense with increasing effective thickness $\delta$, the cluster heights approach the same values as the film thickness for large $\delta>2.5 \mathrm{~nm}$. For small $\delta$, all simulated heights are significantly smaller than the experimental values. For all investigated parameter sets, the difference is about a factor of 2 for very small $\delta$. One reason for this discrepancy might the fact that in the experiment the heights of very thin films were not directly measured, but obtained from an extrapolation of the values for larger $\delta$.

Nevertheless, the origin of this discrepancy remains an open question in this analysis. However, we find that the agreement is improved for larger film thicknesses. Furthermore, we remark that the simulation data does not exhibit any fluctuations in the regime of larger $\delta$ because the histogram which is employed to determine the height becomes more accurate the more particles are added to the system.

Finally, Fig. 3(d) allows us to compare the distances of the clusters. As can be expected, we find a minimum of the distances for small thicknesses $\delta$ that are characterized by a high number density of very small clusters. Although the space between the clusters is decreased by increasing the film thickness $\delta$, the values for the distances increase as they refer to the centers of masses of the clusters. Since in both the experimental and the computational data, the distances and the densities are not determined independently, the evolution of the mean cluster distance exhibits the inverse trends of the density. In particular, the local extrema can be found at the same $\delta$-values. Before the statistical fluctuations set in close to $\delta \approx 2 \mathrm{~nm}$, most simulated distances deviate by less than $5 \%$ from the experimental values. While the limit $\delta \rightarrow 0$ is not resolved by the experimental data, the computational data diverges due to the vanishing cluster density.

\section{Analysis of the influence of the model parameters}

Despite the microscopic treatment of the clusters, the implementation of re-evaporation and trapping processes is only a rough approximation to the real behavior. As a consequence, we conclude by discussing in detail how the corresponding parameters $p_{\text {re }}$ and $\gamma$ affect the evolution of the deposited film. For this analysis, we restrict the maximum thickness to $\delta \approx 1 \mathrm{~nm}$ to make sure that the values are meaningful.

In Fig. 5, the results of a parameter scan are presented for three different values of $\gamma$ (columns I-III) and three different values of $p_{\text {re }}$ indicated by the line styles. For further comparison, we also show the experimental data and add results for the surface coverage which has not been investigated in Ref. [20. Since both the parameters $\gamma$ and $p_{\text {re }}$ have an effect on the sticking of atoms to the surface, it is clear that the influence of these parameters can only be understood by considering both of them together. For example, the influence of the trapping parameter $\gamma$ is relatively weak if the re-evaporation prob- 

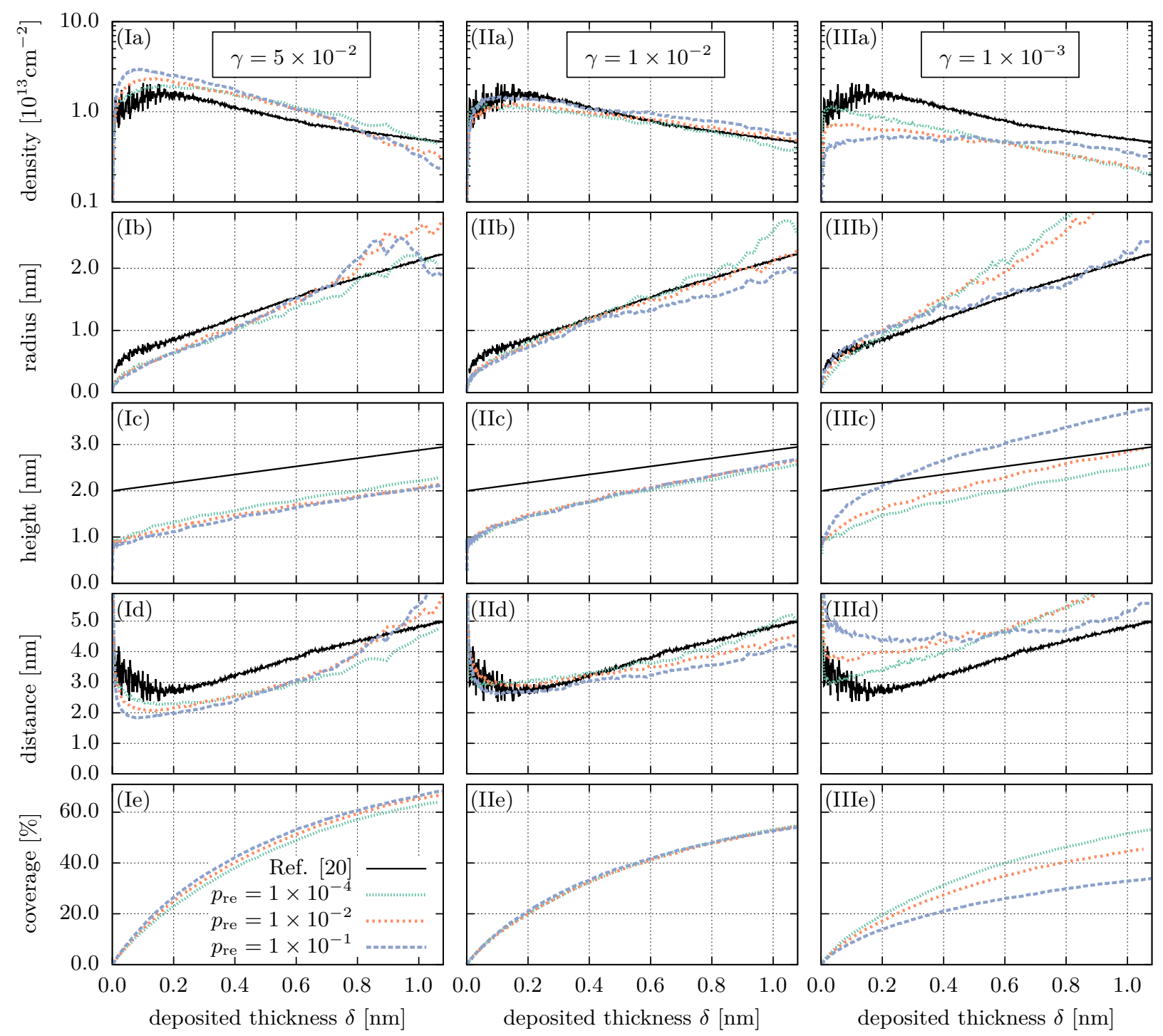

FIG. 5. Evolution of cluster properties (rows 1-4) and surface coverage (row 5) during the deposition process for different fractions of trapped $\mathrm{Au}$ atoms $\gamma$ (represented by each column) and different re-evaporation probabilities $p_{\text {re }}$ (represented by line styles). The black solid curves show the experimental data from Ref. [20].

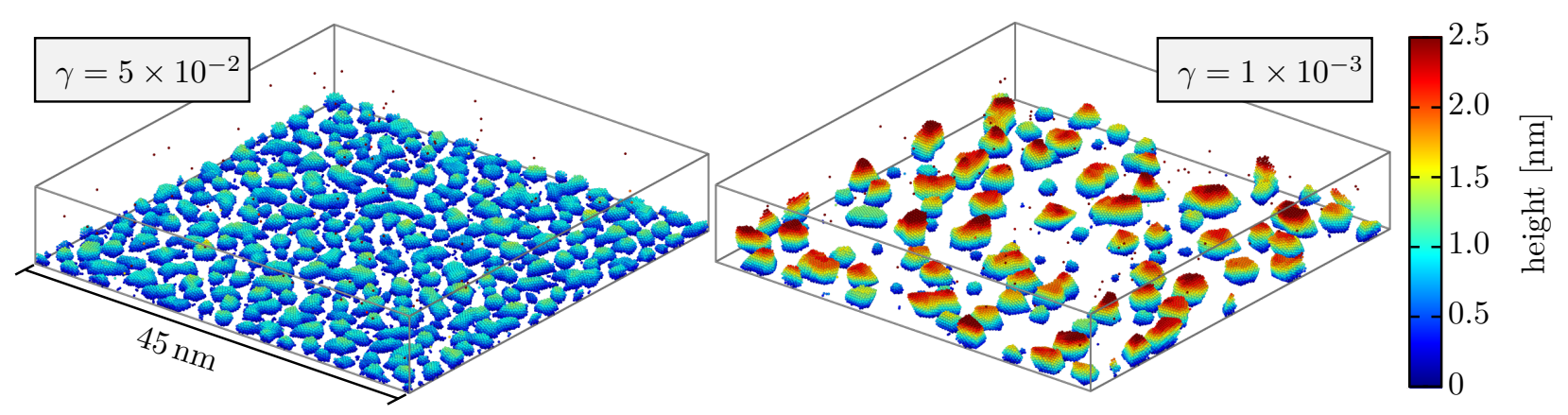

FIG. 6. Simulation snapshots of the film morphologies for different fractions of trapped Au atoms $\gamma$, but equal effective film thicknesses $\delta=0.46 \mathrm{~nm}$. The re-evaporation probability is $p_{\mathrm{re}}=1 \times 10^{-1}$ for both cases. 
ability is small. Hence, the $\gamma$-dependence of all curves with the parameter $p_{\text {re }}=1 \times 10^{-4}$ is not as strong as for the corresponding curves with $p_{\text {re }}=1 \times 10^{-1}$.

A general influence of raising the trapping parameter $\gamma$ is a reduction of the number of diffusing particles, an increased number of nucleation sites and a higher chance that deposited atoms remain on the surface. Consequently, the cluster density (row (a)) can be increased by increasing $\gamma$. This can clearly be observed for the low re-evaporation probability $p_{\mathrm{re}}=1 \times 10^{-1}$ near the maximum values of the density. For further illustration of this trend, we also provide simulation snapshots of films with fixed parameters $\delta=0.46 \mathrm{~nm}$ and $p_{\text {re }}=1 \times 10^{-1}$, but different $\gamma$-values in Fig. 6. Depending on the value of $p_{\text {re }}=1 \times 10^{-1}, \gamma$ affects not only the value of the number density, but also the effective thickness $\delta$ at which the maximum occurs. For $p_{\text {re }}=1 \times 10^{-4}$, it is apparent that a reduction of trapped particles leads to faster coalescence of clusters, which shifts the maximum to smaller values of $\delta$. As a final observation on cluster densities, we point out that there is a trend reversal of the influence of $p_{\text {re }}$ in the third column. If the number of trapped particles is low, the number of clusters on the surface is altered by reducing the re-evaporation probability. However, for $\gamma=5 \times 10^{-2}$ and $\gamma=1 \times 10^{-2}$ the contrary behavior is found. This can be explained by the fact the a high value of the re-evaporation probability hampers the lateral growth and thus the coalescence of the numerous small clusters formed at the defect sites. The numerical data of the cluster radii and heights in rows (b) and (c) confirm this trend. Apart from that, the dependence of these quantities on $p_{\text {re }}$ is weak in the early growth stage. The only exception are the cluster heights for $\gamma=1 \times 10^{-3}$, where a large value of the reevaporation probability is associated with a relatively big cluster height. This is also illustrated in the right snapshot of Fig. 6, where the clusters are particularly high. Since the cluster distance is computed from the number of clusters according to Eq. (8), the values for the distance in row (d) of Fig. 5 mirror all trends that have been described for the density. Finally, in row (e), we also provide data for the surface coverage, which always increases monotonically with $\delta$ and mostly exceeds $50 \%$ for $\delta=1 \mathrm{~nm}$. Only in the case of a small trapping parameter, $\gamma=1 \times 10^{-3}$, where the cluster densities are relatively low, the re-evaporation probability has a noticeable effect on the surface coverage. Although we cannot make a direct comparison with experimental data in this work, we remark that similar experimental results have been reported in Ref. [14, where, for example, a value of $60 \%$ has been measured for the surface coverage at the thickness $\delta=1 \mathrm{~nm}$. This value is close to the value we obtained for $\gamma=1 \times 10^{-2}$ and $\gamma=5 \times 10^{-2}$.

Comparing the cluster properties again to the data from Ref. 20, we find qualitative agreement for most of the data curves. The best quantitative agreement is found for $\gamma=1 \times 10^{-2}$, although none of the parameter combinations removes all discrepancies of the cluster

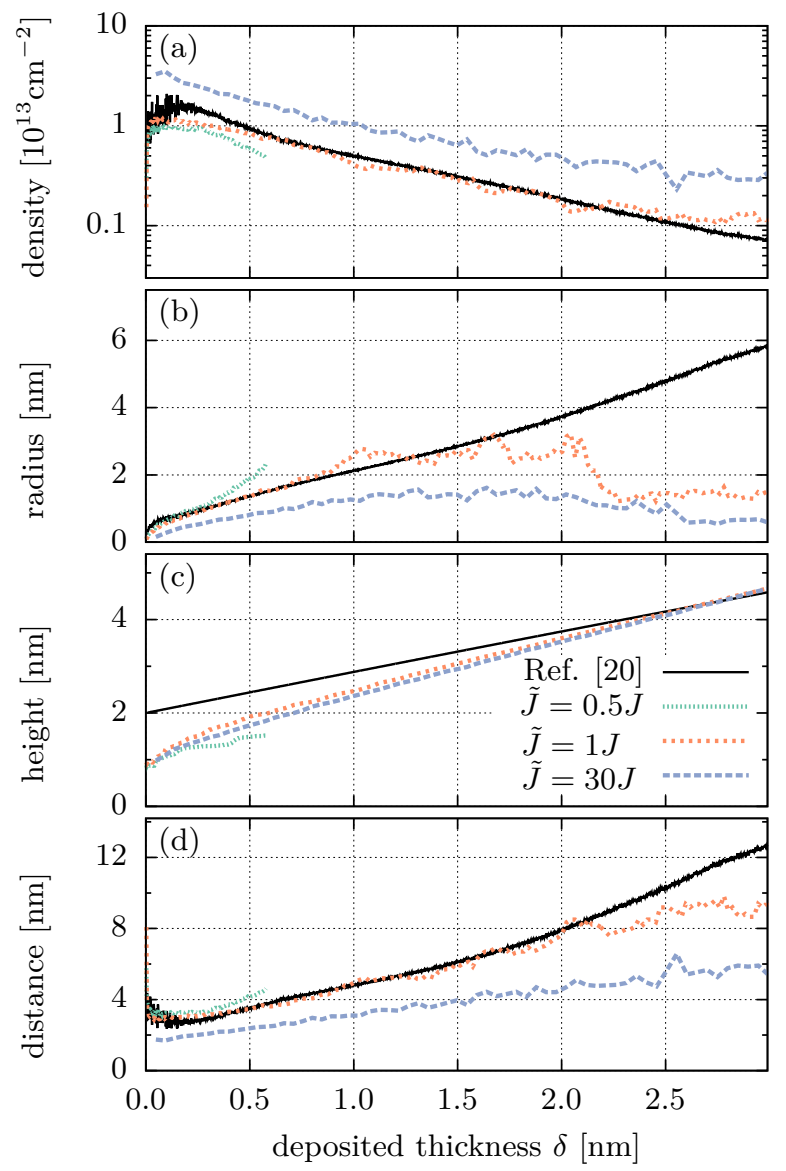

FIG. 7. Cluster properties for different deposition rates $\tilde{J}=s \cdot J$, where the case $s=1$ corresponds to the deposition rate that has been used for all other results in this work. The results have been obtained with the re-evaporation probability $p_{\text {re }}=1 \times 10^{-4}$ and the fraction of trapped $\mathrm{Au}$ particles $\gamma=1 \times 10^{-2}$. For comparison, the experimental data from Ref. 20] (also corresponding to $s=1$ ) are shown by the black solid lines.

heights or maximum cluster densities at once.

\section{E. Influence of the deposition rate}

We conclude the investigation of simulation parameters by analyzing the influence of changing the deposition rate. In Fig. 7, results for the cluster properties are shown for different deposition rates, which are expressed as multiples of the rate $J$ that we used for the other simulations. The simulations were performed with the parameters $\gamma=1 \times 10^{-2}$ and $p_{\text {re }}=1 \times 10^{-4}$, which yielded the best agreement with experimental data in the above analysis.

The plot demonstrates that a high deposition rate, $\tilde{J}=30 J$ leads to higher cluster densities, but similar to what has been found in the investigation of the previous 
section, the clusters have smaller radii, smaller heights and they are closer to each other. The reason for the observed behavior is that a reduction of the cluster number by coalescence is suppressed if the diffusive motion is slow compared to the deposition process. So far, our simulation results do not allow to predict whether these differences will vanish for larger values of $\delta$. Although only high rates are typically of interest for fast processing in technological applications, we also show another curve that represents the simulation with the low rate $\tilde{J}=0.5 J$. Since the corresponding CPU time is roughly twice the time for $\tilde{J}=J$, we only cover a small regime of effective thicknesses. However, this is sufficient to observe trends that are different from the behavior in the case of high rates. As the clusters have more time to move on the surface between deposition events, they have more time to agglomerate. This results in slightly reduced densities and bigger cluster radii. The cluster heights are even smaller than for $\tilde{J}=30 J$, which indicates that the heights scale non-linearly with $\tilde{J}$. The occurrence of reduced cluster heights for small deposition rates can be attributed to relatively long equilibration times in which the cluster radii grow while their heights shrink.

\section{CONCLUSIONS AND OUTLOOK}

In this work, we presented a simulation scheme that can be used to simulate the cluster growth process of sputter-deposited metal atoms on a disordered, fluidlike surface, e.g., a polymer substrate. The simulation model takes advantage of some ideas from previous kinetic Monte Carlo models that have been successfully applied to simulate similar systems [16, 29. These ideas comprise the representation of the substrate as a continuum, which causes random walks of the metal particles, as well as the implementation of simplistic processes that take into account the desorption of atoms on the surface and the creation of defects at the surface by the impingement of highly energetic particles from the plasma. However, while the KMC models rely on simple growth models that neglect atomistic details of the clusters, the new aspect of the present model is the treatment of each individual metal atom with molecular dynamics. Such a microscopic approach allows one to simulate the formation of metallic structures with complicated geometries, which is impossible with the KMC models that require that only spherical or columnar shapes occur.

Compared to KMC simulations, the drawback of this new approach is the limitation of accessible system sizes. While length scales larger than $1 \mu \mathrm{m}$ and time scales in the range of minutes and hours can be accessed with $\mathrm{KMC}$, the scope of this model is restricted to tens of nanometers and the influence of large time scales can only be imitated by a strong proportional increase of the deposition rate and the diffusion coefficient. In the simulations, these quantities are calculated according to Eqs. (3) and (6) after fixing the damping parameter. Al- though the resulting values are up to 9 orders of magnitude larger than typical experimental values, the observed nucleation and coalescence of stable clusters is in accordance with the expected physical behavior and the wide-ranging agreement with experimental data for gold on polystyrene from Ref. [20] supports the significance of the results. Nevertheless, it presently remains difficult to perform benchmarks that go beyond comparisons with experimental results. However, we can state that an upper limit for the damping parameter is given by the value for which the resulting time between the deposition of two atoms is no longer big enough to prevent the atoms from forming clusters above the boundary $z_{\max }$. For the parameter set we used, only atomic deposition with subsequent cluster formation on the surface is observed.

We used the model to investigate how the trapping of metal atoms, the re-evaporation of atoms from the surface and the deposition rate affect the evolution of the cluster morphology. Especially in the early stage of the deposition, specific choices of these parameters may lead to different amounts of mobile surface atoms, which are necessary to trigger cluster coalescence. Consequently, depending on the parameters, one often observes either high densities of clusters with small radii or lower densities of clusters with larger sizes. It can be expected that the influence of the trapping and the re-evaporation becomes less dominant in the case of thick films with high surface coverage, but currently, the simulated film thicknesses are not sufficient to quantify that statement. Reliable statements about the horizontal cluster dimensions can be made as long as most of the clusters do not extend over the periodic boundaries. For the present results, which were obtained with a simulated surface area of $45 \mathrm{~nm} \times 45 \mathrm{~nm}$, this is the case for effective film thicknesses up to $\delta \approx 1.5 \mathrm{~nm}$

While the system sizes we investigated in this work are already sufficient to investigate the nucleation and coalescence of clusters, the significance of this type of simulation can be easily enhanced just by spending more computation time on the simulation of larger segments of the surface and thicker films. Furthermore, a rather comprehensive improvement of the simulation model could include the resolution of individual plasma species, e.g., metal and gas ions, charging of clusters, e.g., by using modified potentials. In particular, experimental or computational studies that yield the fluxes and associated energy distributions of all particle fluxes towards the surface would be of interest to improve the simulations. Another potential application of the simulation scheme is the simulation of the co-deposition processes of, for example, metal and polymer, which could be realized by implementing a continuous shift of the surface. Similar implementations have already been applied in the framework of the aforementioned kinetic Monte Carlo simulations [16, 29.

Finally, we mention that it would also be of interest to simulate the scattering of X-rays at the simulated cluster structures, because this would permit a one-to-one com- 

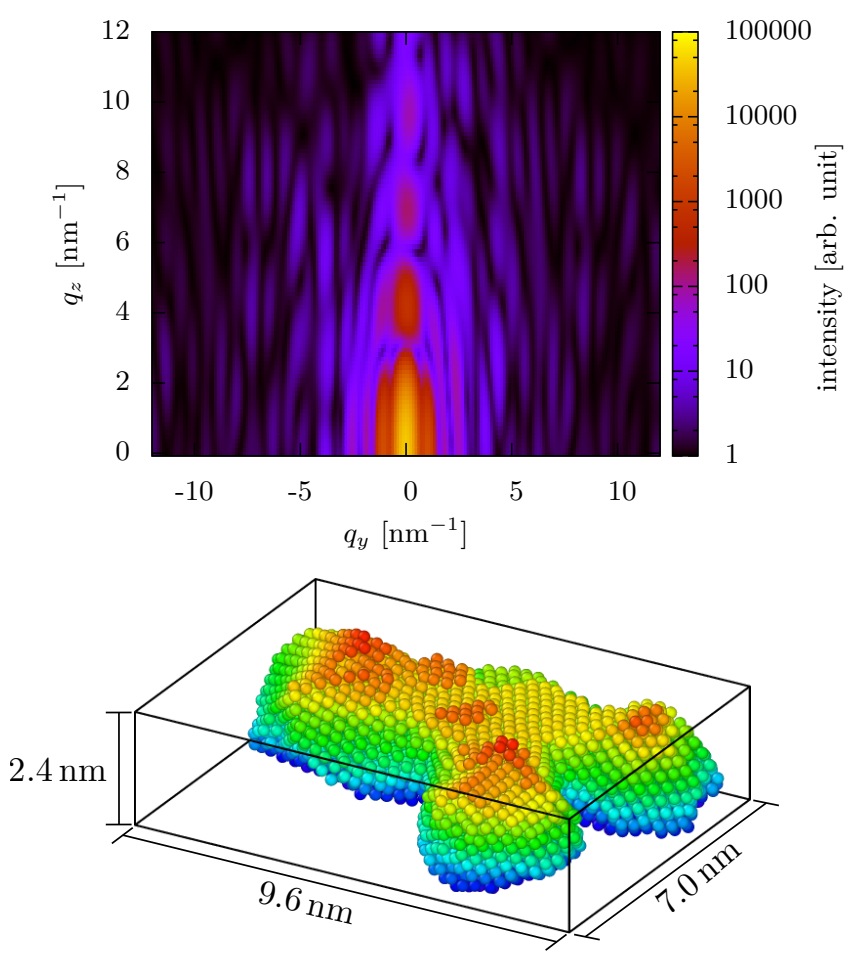

FIG. 8. Scattered intensity (top) of an exemplary cluster (bottom) that is contained in a film with the effective thickness $\delta=1.1 \mathrm{~nm}$.

parison with the quantities that are actually measured in the experiments. Although this is no fundamental problem [54, so far, the surface sizes of the performed simulations are not big enough to generate data with sufficiently small statistical errors. In particular, the interference effects that dominate during the early stages of cluster growth (see Sec. III B cannot be resolved. Yet, even with the existing data, there is another potential application in the field of computational evaluations of GISAXS experiments. As has been described in Sec. IIIB, the real-space data of cluster structures is often obtained by adjusting the properties of simple geometrical objects so that the scattered intensity of the model system fits the measured intensity obtained in the GISAXS measurement. Possibly, one could achieve better fit results by performing the evaluations with more realistic cluster shapes instead of, e. g., hemispheres, cylinders or cubes. For instance, the software BornAgain [55, which is currently being developed, facilitates this, because it includes the functionality to perform the evaluation of GISAXS data with custom cluster shapes. The required input, which could be provided by simulation results, is the Fourier transform

$$
F(\mathbf{q})=\int_{S} \exp (-\mathbf{i q r}) \mathrm{d}^{3} r
$$

of each cluster shape $S$ that shall be included. The inverse length q corresponds to the scattering wave vector. For practical calculations of $F, S$ can be approximated by a set of cuboids. For example we show the scattering features of a single cluster with an irregular shape in Fig. 8, where the scattering cross section has been calculated in simple Born approximation [54, according to

$$
\frac{\mathrm{d} \sigma}{\mathrm{d} \Omega}(\mathbf{q})=F(\mathbf{q}) F^{*}(\mathbf{q})
$$

\section{ACKNOWLEDGMENTS}

This work is supported by the Deutsche Forschungsgemeinschaft via SFB-TR 24 (project A5). We thank Alexander Hinz for discussions of the results and improvements to the manuscript.
[1] T. A. Fulton and G. J. Dolan, Phys. Rev. Lett. 59, 109 (1987)

[2] H. Graf, J. Vancea, and H. Hoffmann, Appl. Phys. Lett. 80, 1264 (2002).

[3] H. Takele, S. Jebril, T. Strunskus, V. Zaporojtchenko, R. Adelung, and F. Faupel, Appl. Phys. A 92, 345 (2008).

[4] M. Karttunen, P. Ruuskanen, V. Pitkänen, and W. M. Albers, J. Electron. Mater. 37, 951 (2008)

[5] A. Biswas, O. Aktas, J. Kanzow, U. Saeed, T. Strunskus, V. Zaporojtchenko, and F. Faupel, Mater. Lett. 58, 1530 (2004).

[6] A. Biswas, O. Aktas, U. Schürmann, U. Saeed, V. Zaporojtchenko, F. Faupel, and T. Strunskus, Appl. Phys. Lett. 84, 2655 (2004).

[7] A. Emamifar, Applications of Antimicrobial Polymer Nanocomposites in Food Packaging, edited by A. Haschim, Advances in Nanocomposite Technology (In-
Tech, 2011).

[8] F.-R. F. Fan and A. J. Bard, J. Phys. Chem. B 106, 279 (2002).

[9] F. Faupel, V. Zaporojtchenko, H. Greve, U. Schürmann, V. S. K. Chakravadhanula, C. Hanisch, A. Kulkarni, A. Gerber, E. Quandt, and R. Podschun, Contrib. Plasma Phys. 47, 537 (2007)

[10] A. Pomogailo and V. Kestelman, Metallopolymer Nanocomposites, edited by R. Hull, R. M. Osgood, Jr., J. Parisi, and H. Warlimont, Springer Series in Materials Science (Springer Berlin Heidelberg, 2006).

[11] G. V. Ramesh, S. Porel, and T. P. Radhakrishnan, Chem. Soc. Rev. 38, 2646 (2009).

[12] Q. Wang and L. Zhu, ACS Symposium Series 1034, Functional polymer nanocomposites for energy storage and conversion (American Chemical Society, 2010).

[13] M. Bonitz, J. Lopez, K. Becker, and H. Thomsen, eds., Complex Plasmas: Scientific Challenges and Technologi- 
cal Opportunities, Springer Series on Atomic, Optical and Plasma Physics, Vol. 82 (Springer-Verlag, 2014).

[14] M. Schwartzkopf, A. Buffet, V. Korstgens, E. Metwalli, K. Schlage, G. Benecke, J. Perlich, M. Rawolle, R. andre, B. Heidmann, G. Herzog, P. Muller-Buschbaum, R. Rohlsberger, R. Gehrke, N. Stribeck, and S. V. Roth, Nanoscale 5, 5053 (2013).

[15] H. Greve, A. Biswas, U. Schürmann, V. Zaporojtchenko, and F. Faupel, Appl. Phys. Lett. 88, 123103 (2006)

[16] L. Rosenthal, H. Greve, V. Zaporojtchenko, T. Strunskus, F. Faupel, and M. Bonitz, J. Appl. Phys. 114, 044305 (2013)

[17] L. Rosenthal, A. Filinov, M. Bonitz, V. Zaporojtchenko, and F. Faupel, Contrib. Plasma Phys. 51, 971 (2011)

[18] V. Zaporojtchenko, T. Strunskus, K. Behnke, C. Bechtolsheim, A. Thran, and F. Faupel, Microelectron. Eng. 50, 465 (2000)

[19] C. Bechtolsheim, V. Zaporojtchenko, and F. Faupel, Appl. Surf. Sci. 151, 119 (1999).

[20] M. Schwartzkopf, G. Santoro, C. J. Brett, A. Rothkirch, O. Polonskyi, A. Hinz, E. Metwalli, Y. Yao, T. Strunskus, F. Faupel, P. Müller-Buschbaum, and S. V. Roth, ACS Appl. Mater. Interfaces 7, 13547 (2015).

[21] F. Faupel, V. Zaporojtchenko, T. Strunskus, and M. Elbahri, Adv. Eng. Mater. 12, 1177 (2010).

[22] U. Schürmann, W. Hartung, H. Takele, V. Zaporojtchenko, and F. Faupel, Nanotechnology 16, 1078 (2005).

[23] D. Depla and S. Mahieu, eds., Reactive sputter deposition, Springer Series in materials science (Springer-Verlag Berlin Heidelberg, 2008).

[24] D. Smith, Thin-Film Deposition: Principles and Practice (McGraw-Hill Education, 1995).

[25] E. Metwalli, S. Couet, K. Schlage, R. Röhlsberger, V. Körstgens, M. Ruderer, W. Wang, G. Kaune, S. V. Roth, and P. Müller-Buschbaum, Langmuir 24, 4265 (2008)

[26] B. M. Smirnov, Cluster processes in gases and plasmas (John Wiley \& Sons, 2009).

[27] D. M. Mattox, J. Vac. Sci. Technol. A 7, 1105 (1989).

[28] L. Rosenthal, T. Strunskus, F. Faupel, J. W. Abraham, and M. Bonitz, Kinetic Monte Carlo Simulations of Cluster Growth and Diffusion in Metal-Polymer Nanocomposites, Chapter in Ref. [13].

[29] J. W. Abraham, N. Kongsuwan, T. Strunskus, F. Faupel, and M. Bonitz, J. Appl. Phys. 117, 014305 (2015).

[30] K.-H. Müller, J. Appl. Phys. 62, 1796 (1987).

[31] C. M. Gilmore and J. A. Sprague, Phys. Rev. B 44, 8950 (1991).

[32] H. Haberland, Z. Insepov, and M. Moseler, Phys. Rev. B 51, 11061 (1995).
[33] L. Dong, R. W. Smith, and D. J. Srolovitz, J. Appl. Phys. 80, 5682 (1996).

[34] R. Rozas and T. Kraska, Nanotechnology 18, 165706 (2007)

[35] L. Xie, P. Brault, C. Coutanceau, J.-M. Bauchire, A. Caillard, S. Baranton, J. Berndt, and E. Neyts, Appl. Catal., B 162, 21 (2014).

[36] A. Thran and F. Faupel, in Defect and Diffusion Forum, Vol. 143 (Trans. Tech. Publ., 1997) pp. 903-910.

[37] M. Bonitz, L. Rosenthal, K. Fujioka, V. Zaporojtchenko, F. Faupel, and H. Kersten, Contrib. Plasma Phys. 52, 890 (2012)

[38] F. Faupel, R. Willecke, and A. Thran, Mat. Sci. Eng. R 22, 1 (1998).

[39] A. Jansen, An Introduction to Kinetic Monte Carlo Simulations of Surface Reactions, Lecture notes in physics (Springer-Verlag, 2012).

[40] S. Plimpton, J. Comp. Phys. 117, 1 (1995)

[41] M. S. Daw and M. I. Baskes, Phys. Rev. B 29, 6443 (1984).

[42] S. M. Foiles, M. I. Baskes, and M. S. Daw, Phys. Rev. B 33, 7983 (1986)

[43] Depending on the plasma parameters and thermal broadening, the deposited particles may have different kinetic energies. This only has a weak influence as long as no resputtering occurs or defects in the polymer are created. The latter effect is taken into account by a separate process in the model.

[44] T. Schneider and E. Stoll, Phys. Rev. B 17, 1302 (1978).

[45] B. Dünweg and W. Paul, Int. J. Mod. Phys. C 2, 817 (1991)

[46] V. Zaporojtchenko, K. Behnke, T. Strunskus, and F. Faupel, Surface and Interface Analysis 30, 439 (2000).

[47] V. Zaporojtchenko, K. Behnke, A. Thran, T. Strunskus, and F. Faupel, Appl. Surf. Sci. 144, 355 (1999).

[48] R. V. Stuart, G. K. Wehner, and G. S. Anderson, J. Appl. Phys. 40, 803 (1969).

[49] V. Georgieva, M. Saraiva, N. Jehanathan, O. I. Lebelev, D. Depla, and A. Bogaerts, J. Phys. D: Appl. Phys. 42, 065107 (2009)

[50] A. Stukowski, Modelling and Simulation in Materials Science and Engineering 18, 015012 (2010).

[51] L. Bai and D. Breen, Journal of Graphics, GPU, and Game Tools 13, 53 (2008).

[52] J. R. Levine, J. B. Cohen, Y. W. Chung, and P. Georgopoulos, J. Appl. Crytallogr. 22, 528 (1989)

[53] J. R. Levine, J. B. Cohen, and Chung, Surf. Sci 248, 215 (1991).

[54] R. Lazzari, J. Appl. Cryst. 35, 406 (2002).

[55] C. Durniak, M. Ganeva, G. Pospelov, W. V. Herck, and J. Wuttke, "BornAgain - Software for simulating and fitting X-ray and neutron small-angle scattering at grazing incidence, version 1.4," http://www.bornagainproject. org/ (2015). 